Arthroskopie $2019 \cdot 32: 165$

https://doi.org/10.1007/s00142-019-0274-6

(c) Springer Medizin Verlag GmbH, ein Teil von Springer Nature 2019

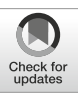

\author{
P. Niemeyer ${ }^{1,2} \cdot$ Andreas B. Imhoff $^{3}$ \\ 'Orthopädische Chirurgie München (OCM), München, Deutschland \\ ${ }^{2}$ Klinik für Orthopädie und Unfallchirurgie, Universitätsklinikum Freiburg, Freiburg im Breisgau, \\ Deutschland \\ ${ }^{3}$ Abteilung für Sportorthopädie, Klinikum rechts der Isar, Technische Universität München (TUM), \\ München, Deutschland
}

\title{
Regenerative und biologische Verfahren am Kniegelenk
}

gel vom Kollagen-Typ II (CTX-II) aufweisen als die nicht mit einem Kortikosteroid behandelten Placebogruppen. Dies ist ein weiteres Argument, dass eine posttraumatische Gonarthrose mit dem Zeitpunkt der Verletzung beginnt und zu einer dramatischen Veränderung der Knorpelmatrix führen kann [1].

Für den klinisch tätigen Orthopäden in Deutschland gilt die Anwendung von Zell- und Gewebeaufbereitungen zum jetzigen Zeitpunkt als problematisch, und die Regulation erscheint uneinheitlich und unübersichtlich. Die intraartikuläre Zellapplikation ist hier nach aktueller Ansicht der Behörden als Arzneimitteltherapie anzusehen, weshalb zurzeit am ehesten eine Anwendung von Fettgewebsaufbereitungen mit Applikation nicht in das Gelenk als „außerhalb der ATMPVerordnung" realisierbar und nach Anzeige beim Regierungspräsidium durchführbar ist [2].

Das vorliegende Themenheft „Regenerative und biologische Verfahren am Kniegelenk“ widmet sich umfassend den unterschiedlichen aktuellen Entwicklungen und Konzepten der Therapie von Knorpeldefekten. In diesem Sinne wünschen wir Ihnen eine lehrreiche, aber auch unterhaltsame Lektüre der aktuellen Ausgabe der Arthroskopie und hoffen, mit praktischen Tipps und Hinweisen Ihre klinische Tätigkeit bereichern zu können.

Prof. Philipp Niemeyer und Univ. Prof. Andreas B. Imhoff München, 11.03.2019

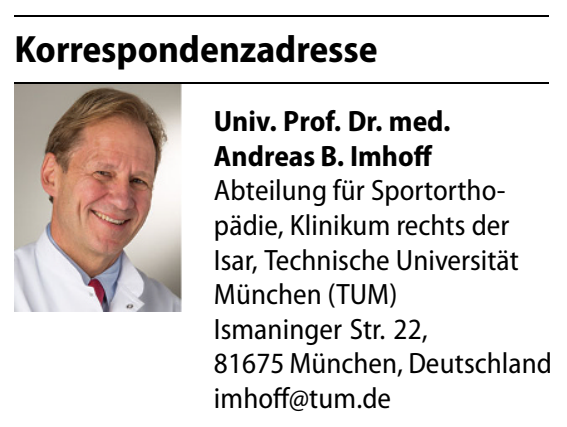

Interessenkonflikt. P. Niemeyer und A. Imhoff geben an, dass kein Interessenkonflikt besteht.

\section{Literatur}

1. Lattermann C, Jacobs CA, Proffitt Bunnell M, Huston LJ, Gammon LG, Johnson DL, Reinke EK, Huebner JL, Kraus VB, Spindler KP (2017) A Multicenter study of early anti-inflammatory treatment in patients with acute anterior cruciate ligament tear. Am J Sports Med 45:325-333

2. Eigenfettbehandlung $S$ (2019) Wenn Gewebe zur Arznei wird.Dtsch Arztebl 116:28-30 